\title{
Midiendo la calidad de la información gestionada: algunas reflexiones conceptuales-metodológicas
}

\author{
Carlos Luis González-Valiente \\ Grupo Empresarial de la Industria Sidero-Mecánica - GESIME, Cuba
}

\section{REVIEW}

\begin{abstract}
Resumen
El estudio, basado en un análisis documental clásico, reflexiona sobre algunas directrices conceptuales-metodológicas relativas a la medición de la calidad de la información (Cl) en el marco de la gestión de información (GI) en las organizaciones. Es descrito el proceso de Gl y la importancia de la aplicación de principios de calidad en éste. Se exponen las cuatro dimensiones de la $\mathrm{Cl}$ según una integración de los indicadores esenciales que caracterizan a los contenidos informacionales. Son definidas cada una de las fases que componen el diseño de una metodología para evaluar la información. Se indican las implicaciones que tiene este ejercicio para los profesionales del campo informacional.
\end{abstract}

Palabras clave

Calidad de información ; Gestión de información ; Dimensiones de calidad de información ; Metodología de calidad de información

\section{Measuring the quality of information managed: some conceptual and methodological reflexions}

\begin{abstract}
The study, based on a documental classic analysis, presents conceptual and methodological guidelines concerning the design of methodologies that help to measure the quality of information that is managed in organizations. It is described the process of information management and the importance of implementing quality principles in it. There are exposed the four dimensions of information quality as part of an indicators integration which characterize the informational contents. There are defined each of the phases in the methodological design to evaluate the information. There also are indicated the implications of this activity for information professionals.
\end{abstract}

Keywords

Information quality ; Management of information ; Information quality dimensions ; Information quality methodology

\section{Introdución}

Investigaciones enfocadas en las temáticas de $\mathrm{Cl}$ se concentran mayormente en cuestiones de calidad desde la perspectiva de los sistemas, productos o servicios. Esto lo corrobora una búsqueda exploratoria en la base de datos ScienceDirect (1), en la cual bajo la ecuación de búsqueda Information Quality en el título del conjunto de revistas indizadas, fueron determinados como tópicos más abordados: internet, sitio web, calidad de servicio, sistema de información y tecnología de información.

Es importante comprender que la determinación de la $\mathrm{Cl}$ es una actividad implícita en procesos que son puramente informacionales. En el contexto específico de la Gl en las organizaciones, ésta como actividad informativa bien 
integradora, la $\mathrm{Cl}$ es crucial porque las decisiones dependen de la buena formulación que los profesionales de la información sean capaces de ejecutar (Ballou \& Tayi, 1999).

Este artículo pretende reflexionar sobre algunas directrices conceptuales-metodológicas relativas a la medición de la $\mathrm{Cl}$ en el marco de la Gl en el dominio organizacional. Mediante un análisis de algunas investigaciones relevantes que han abordado esta temática se delimitarán los elementos básicos en el diseño de enfoques metodológicos sobre $\mathrm{Cl}$. Por último, se enunciarán las implicaciones que tiene el ejercicio de esta actividad para los profesionales del campo informacional, con la finalidad de destacar pistas esenciales para el mejoramiento de sus tareas de trabajo.

\section{La información y su proceso de gestión}

Antes de comenzar deconstruyendo cada una de las categorías de análisis de este estudio se precisará conceptualmente que es la información, debido a las diferentes interpretaciones que le son conferidas. De forma general se asocia información a dato y a conocimiento; y aunque esta tríada presenta una gran interrelación, la naturaleza de sus individuales significados es muy cuestionable (Zins, 2007). Disparmente, la literatura sobre gestión lo vincula más conocimiento (Bates, 2006), mientras que los abordajes específicos sobre $\mathrm{Cl}$ lo relacionan al dato, debido al vínculo directo de esta temática con los sistemas tecnológicos. El interés de este artículo es explorarla desde la perspectiva en la que ésta puede ser gestionada, por ello se paraleliza información y dato, como elementos que, bajo profundos parámetros de análisis, llenan vacíos de conocimiento que permiten llevar a cabo o redirigir acciones concretas.

La Gl, ésta es considerada por Ponjuán (2011) como la actividad o función estratégica que concreta las políticas de información de las instituciones. Más estructuralmente, Choo (1997) la operacionaliza en una red de seis procesos interrelacionados: identificación de necesidades de información, adquisición de información, organización y almacenamiento de información, desarrollo de productos y servicios de información, y diseminación y uso de la información. Ellos son delimitados a partir de la premisa de que las organizaciones se comportan como un sistema abierto que transforma la información en conocimientos, procesos y estructuras que generan, a su vez, nuevos productos y servicios (Choo, 1996). En dicha transformación están implícitas actividades claves como la determinación de la calidad de los contenidos informacionales, considerado por Ponjuán (2002) uno de los elementos que integra el alcance de la Gl en el entorno organizacional.

La materialización de la información como un recurso crítico para la toma de decisiones y la gerencia (Alwis de \& Higgings, 2001) presupone que la calidad de la planeación estratégica dependa de la calidad de los recursos informativos adquiridos tras el análisis de las numerosas fuentes disponibles (Auster \& Choo, 1994). Sin embargo es importante señalar algo que Ponjuán (2011) precisa conceptualmente cuando valora los modelos de Gl de Butcher y Rowley (1998), Paéz Urdaneta (1992), Choo (1992) y Ponjuán (2000). No debe confundirse este concepto cuando se aborde "lo referido al acceso y uso de la información o a su tratamiento, procesamiento y organización" (p.16). Cada una de estas tareas puede darse independientemente una de otra y caer perfectamente bajo los marcos conceptuales del comportamiento informacional (2) que las personas asumen a la hora de manejar la información, lo cual no implica que se esté hablando en términos puramente de GI. Ponjuán (2011) distingue que esta actividad debe concebirse y manejarse como una función estratégica institucional y no como proceso operativo que aseguran el tratamiento de la información y la prestación de servicios.

\section{Dimensiones de la calidad de la información}

Peter (2011) afirma que la información debe poseer calidad para que sea considerada como información. Precisamente, muchos autores consideran que la información posee calidad cuando ésta es completamente útil en la toma de decisiones o la solución de algún problema específico. Por lo que si el proceso que conlleva a la determinación de la calidad informativa falla, la decisión tomada o el problema a solucionar no serán factibles, ya que un factor es condicionante preciso del otro. Esto tiene lugar, en gran medida, por la marcada subjetividad subyacente en cada una de las estructuras mentales de quienes encaminan esta actividad. Aquí los patrones, modelos y estructuras cognitivas definen, debido a que las personas no solo crean construcciones subjetivas de su 
experiencia, sino que esas construcciones de la información también tienen una existencia objetiva en el sistema nervioso y son externalizadas en acciones específicas (Choo, 1996).

Van Birgelen, De Ruyter y Wetzels consideran, similarmente, que la utilización de la información de calidad está determinada por el nivel de satisfacción del proceso y resultado de un proyecto de investigación específico (2001). El gráfico 1 representa a dicha satisfacción como el componente de núcleo de cualquier proyecto.

\section{Gráfico 1 - Marco conceptual del uso de la información relacionada com la calidad}

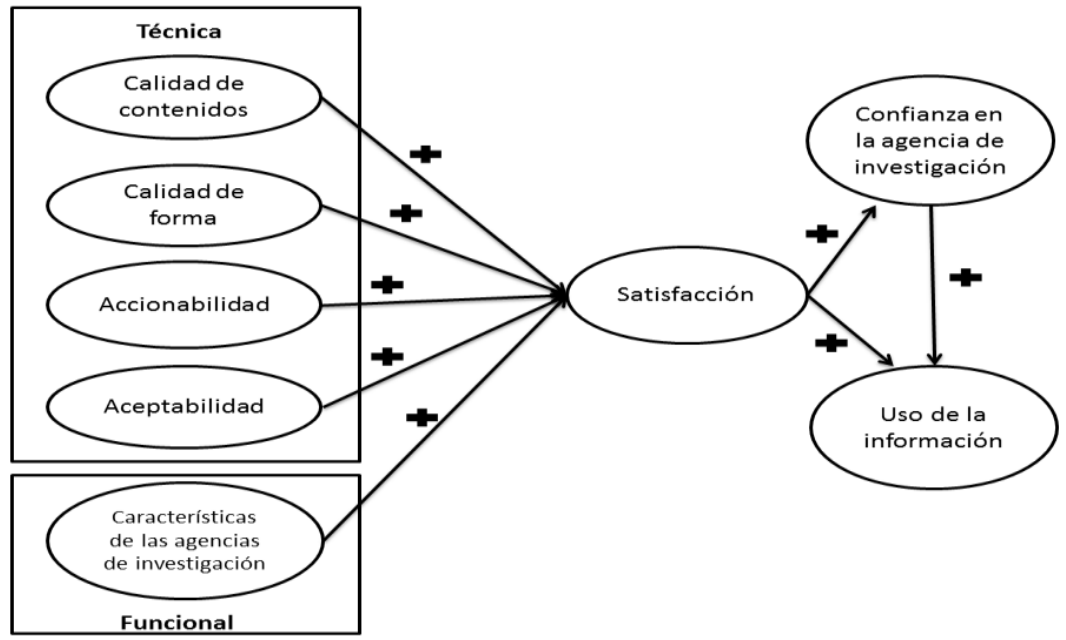

Fuente: Van Birgelen, De Ruyter \& Wetzels, 2001.

La marcada subjetividad implícita en un proceso de $\mathrm{Cl}$ ha impulsado la multiplicidad de variables que hacen factible su determinación y evaluación. Su variabilidad viene dada mayormente por la relación objeto de informacióncontexto de información, es decir, una proporcionalidad entre contenido-forma y el contexto situacional. En ese contexto es donde entrarían agentes como las capacidades de las personas, los requerimientos de la organización, los procesos asociados, etc. Para Stvilia (2007) el contexto posee dos componentes básicos: la cultura (lenguaje, normas) y las estructuras sociotécnicas (incluyendo relaciones económicas y estándares). Del objeto de información es determinante la particularidad o atributo específico que es importante medir.

Las dimensiones son uno de los principales componentes de la Cl, y éstas son agrupadas por Lee et al. (2008) en cuatro categorías: intrínseca, contextual, representacional y acceso (Véase tabla 1). Estas categorías han sido previamente definidas por Strong (1997), quien las considera como las que mayor calidad le atribuyen a la información. Para estos autores la dimensión intrínseca concibe a la información como algo que tiene calidad por derecho propio. Mientras que la contextual destaca que los requerimientos de calidad se dan en un contexto dado y que la información tiene que ser entonces relevante, oportuna, completa y apropiada en términos de cantidad; así como de agregación de valor. Por último, las dimensiones representacional y de acceso enfatizan la importancia de los sistemas de cómputo para el almacenamiento y el acceso de la información. 
Tabla 1. La mirada académica de la calidad de la información (Continua)

\begin{tabular}{|c|c|c|c|c|c|}
\hline & Intrínseca & Contextual & Representacional & Acceso & \\
\hline $\begin{array}{l}\text { Wang and Strong } \\
\text { (1996) }\end{array}$ & $\begin{array}{l}\text { Precisión, } \\
\text { credibilidad, } \\
\text { reputación, } \\
\text { objetividad }\end{array}$ & $\begin{array}{l}\text { Valor añadido, } \\
\text { pertinencia, } \\
\text { completa, } \\
\text { oportunidad, } \\
\text { cantidad apropiada }\end{array}$ & $\begin{array}{l}\text { Entendible, } \\
\text { interpretable, } \\
\text { representación } \\
\text { concisa, } \\
\text { representación } \\
\text { consistente }\end{array}$ & $\begin{array}{l}\text { Accesibilidad, } \\
\text { facilidad } \\
\text { operaciones, } \\
\text { seguridad }\end{array}$ & de \\
\hline Zmud (1978) & Precisa, factual & $\begin{array}{l}\text { Cantidad, confiable / } \\
\text { tiempo }\end{array}$ & $\begin{array}{l}\text { Disponible, leíble, } \\
\text { razonable }\end{array}$ & & \\
\hline $\begin{array}{l}\text { Jarke and } \\
\text { Vassiliou (1997) }\end{array}$ & $\begin{array}{l}\text { Creíble, veracidad, } \\
\text { credibilidad, } \\
\text { coherencia, } \\
\text { completa }\end{array}$ & $\begin{array}{lr}\text { Relevancia, } & \text { uso, } \\
\text { oportuna, circulación } \\
\text { de la ruente, } \\
\text { circulación r de } \\
\text { almacén de datos, } \\
\text { no volátil } & \end{array}$ & $\begin{array}{l}\text { Interpretabilidad, } \\
\text { sintaxis, } \\
\text { control de versiones, } \\
\text { semántica, } \\
\text { renombrada, origen }\end{array}$ & $\begin{array}{l}\text { Accesibilidad, } \\
\text { disponibilidad } \\
\text { sistema, } \\
\text { disponibilidad } \\
\text { transacción, } \\
\text { privilegios }\end{array}$ & $\begin{array}{l}\text { del } \\
\text { de }\end{array}$ \\
\hline
\end{tabular}

Fuente: Lee et al., 2008.

Tabla 1. La mirada académica de la calidad de la información (Conclusión)

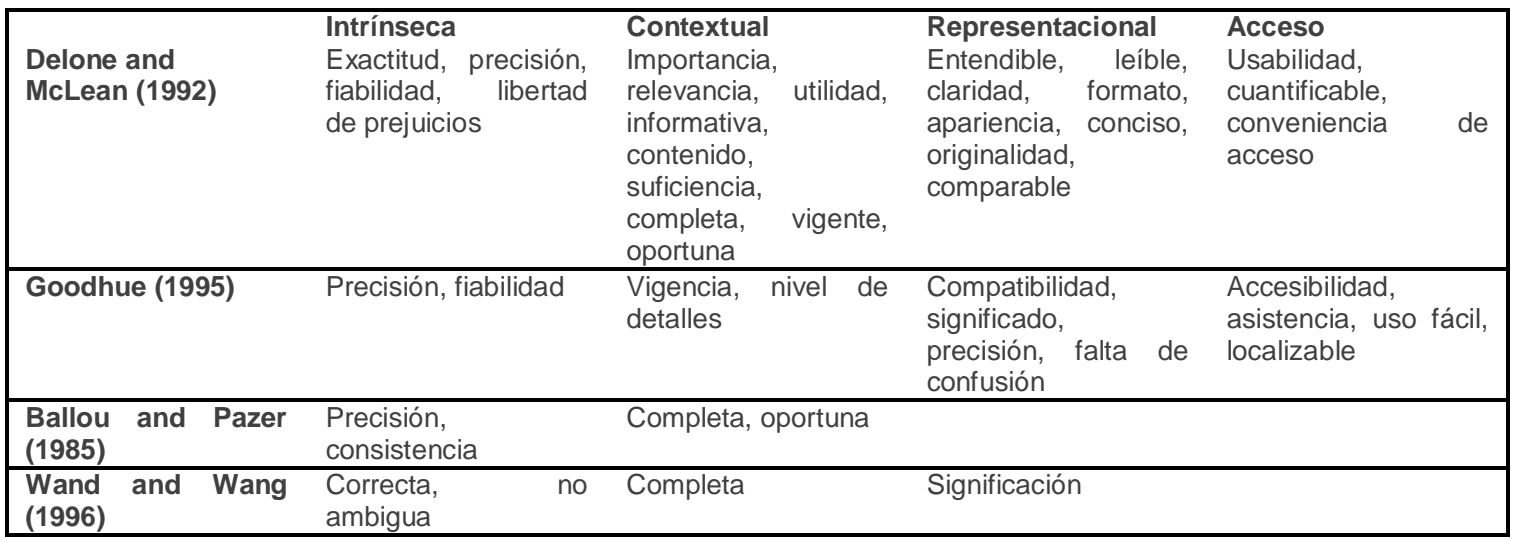

Fuente: Lee et al., 2008.

En estas dimensiones, nuevas propuestas han sido expuestas en la literatura científica, como es el caso de Ruby y Vashchilko (2012), quienes sugieren la veracidad/decepción (3), como atributo que debe incluirse en la categoría intrínseca. Cada dimensión funciona como un marco conceptual y de referencia para la modelación de la cualificación informativa. Pues aquí se integran las variables apropiadas que fungen como objeto para la ejecución de las evaluaciones apropiadas. Según Stvilia et al. (2007) "el marco de evaluación de la $\mathrm{Cl}$ como estructura multidimensional consiste en conceptos, relaciones, clasificaciones y metodologías generales que pudieran servir como un recurso o guía para desarrollar modelos de medición de $\mathrm{Cl}$ en un contexto específico" (p.1722).

\section{Procedimiento para la determinación y medición de la calidad informativa}

Múltiples perspectivas son tomadas para elaborar enfoques metodológicos que ayudan a determinar la calidad de los contenidos informacionales que diariamente se gestionan. Entre las nueve perspectivas existentes se destacan las enunciadas por Betini et al. (2009): 
1. Las fases y los pasos que componen la metodología

2. Las estrategias y los métodos para medir o evaluar la información

3. Las dimensiones y las metrías adoptadas

4. Los tipos de costos asociados

5. Los tipos de datos que son considerados

6. Los tipos de sistemas de información que usan, modifican y manejan los datos

7. Las organizaciones incluidas en los procesos que crean o eliminan los datos

8. Los procesos que crean o eliminan los datos que tributan a la producción de servicios

9. Los servicios que son producidos por los procesos considerados en la metodología

La multiplicidad de estas metodologías varía según el contexto de aplicación, los procesos asociados y los resultados a los que dichos procesos tributan. Capiello y Pernici (2006) y Batini et al. (2009) coinciden en que son cuatro las fases que la organización debe comprender para determinar dicha calidad; éstas son: la definición de la calidad de los datos, la medición, el análisis y el mejoramiento. En respuesta a los planteamientos de estos autores, en este estudio solo se tendrán en cuenta las tres primeras perspectivas enunciadas anteriormente.

\subsection{Definición de la calidad de la información}

En esta primera se recolecta toda la información y se definen los indicadores a medir, teniendo en cuenta cada una de las cuatro categorías que componen las dimensiones de calidad expuestas por Lee et al (2008). Es necesario, constar de una exploración o análisis previo del entorno en el cual la información se encuentra o transita. Puede que sea, por ejemplo, un sitio web o una red social, un sistema de información implementado, un sistema de almacenamiento de datos que la organización posea o una base de datos.

Para ello es necesario identificar el proceso organizacional en el cual la medición se enmarca, en correspondencia con los requerimientos de información de los usuarios (empresa, departamento, equipo de trabajo especializado, especialista individual). Strong (1997) considera que la Cl no puede ser medida independientemente de las personas que la usan, donde la mirada, además de dirigirse a los usuarios o consumidores, debe dirigirse también a los productores y consumidores de información.

\subsection{Medición de la calidad de la información}

Los métodos para medir o evaluar la calidad de la información son usados de acuerdo a los resultados que se deseen obtener. Como mayormente esta es una actividad que está condicionada por la perspectiva subjetiva de los individuos (percepción, razonamiento, interpretación), es factible la combinación de técnicas cuantitativas (análisis numéricos y estadísticos) y cualitativas (entrevistas, cuestionarios). Por ejemplo, Lee et al. (2008) diseñaron un cuestionario y aplicaron un muestreo para medir la $\mathrm{Cl}$ en una organización; para ello delimitaron ítems que respondían a cada una de las categorías de las dimensiones antes mencionadas. A cada uno de estos ítems les fue asignada una escala de valor con la cual se determinó el comportamiento de cada variable dentro de las dimensiones. Por otro lado, Eppler y Muenzenmayer (2002) han expuesto métodos para el entorno específico de la web, entre los cuales destacan los siguientes:

a) El monitoreo de rendimiento 
b) El analizador de sitio

c) El analizador de tráfico

d) La minería web

e) La retroalimentación del usuario

En los argumentos de su investigación afirman que de estas cinco tipologías existen software disponibles, específicamente para el tipo a), b) y c). Mientras que para d) y e) hay herramientas más poderosas pero muy costosas. Ahora, lo que sí varía en estas cinco tipologías es la presencia de atributos de la información sobre los cuales no es posible aplicar cualquier tipo de variable. Además, que dicha tipología responde más al medio (forma) que al modo (contenido) de la información; aunque ambas cuestiones son importantes porque resulta difícil desliar un elemento del otro. De ahí la relevancia de identificar previamente cuáles son los parámetros de calidad que se desean evaluar, según lo expuesto en la tabla 1. Es oportuno destacar que sobre esta temática muchas de los estudios emergidos de la literatura exponen evaluaciones a partir de ecuaciones algebraicas u otra clase de análisis desde la perspectiva de los sistemas tecnológicos y procesos de software (ej. Su \& Yi, 2008; Caballero, Caro \& Piattini, 2008).

\subsection{Análisis del resultado de la medición}

Para la fase de análisis hay autores que abogan por la categorización de los elementos obtenidos (ej. Fink-Shamit \& Bar-llan, 2008); en este caso referido a los atributos incluidos en cada dimensión (Véase tabla 1). A cada grupo de elementos se le puede asignar un valor tanto numérico como descriptivo. En esta tarea pueden darse niveles de participación múltiple o individual para analizar los resultados obtenidos, en donde los especialistas emiten su criterio para identificar los patrones emergentes durante el proceso evaluativo. Un modelo simple para ello puede ser el propuesto en la tabla 2, en donde convergen externamente el proceso específico de Gl y el requerimiento implantado por la organización. Estos requerimientos aquí se traducen como algo circunstancial; y no son más que un problema dado o una necesidad expresa sobre la cual la GI se está enfocando. Dicha GI puede orientarse tanto a un proceso organizacional (investigación de mercado, análisis del entorno, inversiones, transacciones, etc.), como a un servicio o producto, según la fase de desarrollo o análisis de éstos. Sobre ellos serán distinguidos y descompuestos los elementos estructurales que lo integren como lo ejemplifica la tabla 2. En la Dimensión de la Cl se expondrán los atributos a los cuales se les añadirá un valor deseado y una breve descripción del resultado de la evaluación.

Tabla 1. Modelo para medir la calidad de la información

Especificación del proceso de GI

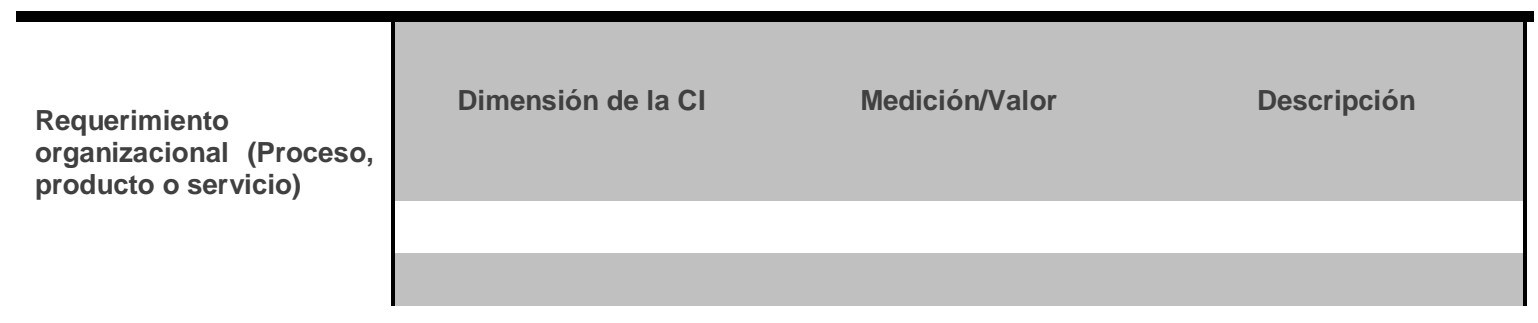

Fuente: Elaboración propia 


\subsection{Mejoramiento de la calidad de la información}

El mejoramiento de la información es una de las etapas que se tiene en cuenta cuando la finalidad del proceso de $\mathrm{Cl}$ va dirigida a un producto, servicio o sistema de información específico. Ello incide en que las prácticas de gestión sean replanteadas y por lo tanto se desecha o acepta la información utilizada. Caballero et al. (2008) afirman que el primer paso es realizar una evaluación del escenario en el cual la mejora se realizará (negocios, web, software, sistemas de gestión), aunque actualmente no existen muchos modelos de referencia que permitan demostrar cuán optimo un escenario puede ser. Batini et al (2009) destacan que en esta etapa se definen las medidas, estrategias y técnicas para alcanzar los nuevos objetivos de calidad de la información.

\section{Implicaciones profesionales}

Si se ha estado hablando de la necesidad de aplicar enfoques de $\mathrm{Cl}$ en el proceso de $\mathrm{Gl}$, es clave identificar como nexo la perspectiva humana, la de los profesionales de la información. Ballou y Tayi (1999) confirman que los gestores de la información, en conjunto con los usuarios de ésta, deben pensar sistemáticamente en función de lo que es necesario, vital y deseado. Además de que estas iniciativas deben estar guiadas de acuerdo a las ideas, políticas y tendencias de $\mathrm{Cl}$ que están presentes en la organización. Esto encuadra perfectamente con lo enunciado por Ponjuán (2011), cuando enuncia que la GI se materializa en términos de políticas de información específicas. Sin embargo desde una perspectiva inversa, o como una forma de contribución, Caballero et al (2008) indican que las responsabilidades de estos especialistas van encaminadas a determinar las políticas de $\mathrm{Cl}$ según los requerimientos organizacionales, de manera tal que se puedan identificar de forma certera las técnicas, herramientas y procedimientos para evaluar el recurso información. Siempre teniendo como base el contexto en el cual ésta transita y se usa. Pues ello contribuiría a definir modelos conceptuales y estratégicos según los procesos específicos a los que la Gl se orienta.

\section{Consideraciones finales}

La gestión constante de información es una práctica que debe estar condicionada por la aplicación de parámetros de calidad que permitan que la información suministrada, compartida y usada posea los requisitos óptimos. Actualmente son muchas las metodologías que existen, las cuales difieren en dependencia del contexto organizacional que se aplica. Dicho contexto está básicamente definido por los procesos y las personas implícitas en ellos. Esto conduce a la construcción de modelos de calidad de información que responden a entornos específicos, los cuales deben tener como base conceptual cada una de las dimensiones de calidad informativa (intrínseca, contextual, representacional y accesible) declaradas. Los parámetros metodológicos que aquí se resumen están mayormente dirigidos al desarrollo de sistemas; pero aplica de igual forma a todo tipo de prácticas de Gl, porque esta actividad debe atender a los componentes del mismo y a su vez optimizarlo de manera que se puedan ejecutar efectivamente las funciones de gestión (Ponjuán, 2011).

Es bueno considerar que cualquier práctica informativa requiere de garantías de calidad, lo cual presupone que los profesionales de la información se responsabilicen por los procesos de producción informativa a través de cada una de las etapas del ciclo de gestión de información a los que Choo (1996) hace alusión. Pues estos especialistas fungen como filtros humanos que, mediante conocimientos y habilidades oportunas, inciden en la calidad dada en la relación establecida entre el usuario del sistema y la información provista.

\section{Notas}

1. ScienceDirect, en su sitio web, se presenta como una de las bases de datos científica de la compañía Elsevier, la cual ofrece acceso a artículos de revistas y capítulos de libros que han sido previamente arbitrados. Posee además una infraestructura adecuada para la ejecución de búsquedas que son oportunas para el análisis bibliométrico de los contenidos (http://info.sciencedirect.com/about). 
2. El comportamiento informacional estudia "cómo las personas necesitan, buscan, comparten y usan la información en diferentes contextos, incluyendo el lugar de trabajo y la vida diaria" (Pettigrew, Fidel \& Bruce, 2001, p.45).

3. Para mayor profundidad de lo que se propone desde este componente en la $\mathrm{Cl}$, consúltese a Ruby y Vashchilko (2012).

\section{Bibliografía}

Auster, E., \& Choo, C. W. (1994). How senior managers acquire and use information in environmental scanning. Information Processing \& Management, 30 (5), 607-618.

Ballou, D. \& Tayi, G. (1999). Enhancing data quality in data warehouse environments. Communications of the ACM; 42 (1), 73-78. Recuperado de: http://www.atilim.edu.tr/ ccaydin/word powerpoint/ex62.pdf

Bates, M. J. (2006). Fundamental forms of information. Journal of the American Society for Information Science and Technology, 57(8), 1033-1045.

Batini, C., Cappiello, C., Francalanci, C. \& Maurino, A. (2009). Methodologies for data quality assessment and improvement. ACM Computing Surveys (CSUR); 41 (3). Recuperado de: http://cat.inist.fr/?aModele=afficheN\&cpsidt=21901796

Caballero, I., Caro, A., Calero, C. \& Piattini, M. (2008). IQM3: Information quality management maturity model. Journal of Universal Computer Science, 14(22), 3658-3685. Recuperado de:

http://www.researchgate. net/publication/220348856 IQM3 Information Quality Management Maturity Model/file/9fcfd50cb913a2d $\underline{\text { c02.pdf }}$

Cappiello, C. \& Pernici, B. (2006). A methodology for information quality management in self-healing web services. International Conference on Information Quality; Noviembre; Boston. Recuperado de: http://home.deib.polimi.it/pernici/papers/PaperlQ2006CappielloPernici.pdf

Choo, C. W. (1996). The knowing organization: how organizations use information to construct meaning, create knowledge and make decisions. International Journal of Information Management, 16 (5), 329-340. Recuperado de:

http://zaphod.mindlab.umd.edu:16080/docSeminar/pdfs/Choo1996.pdf

Choo, C. W. (1997). Organizations as "information-use systems": a process model of information management. Universiteit van Amsterdam, Department of Information Management. Recuperado de: http://primavera.fee.uva.nl/PDFdocs/97-17.pdf

Alwis de, S. M. \& Higgins, SE (2001). Information as a tool for management decision making: A case study of Singapore. Information Research, 2(7), 7-1. Recuperado de: http://informationr.net/ir/7-1/paper114

Eppler, M. J. \& Muenzenmayer, P. (2002). Measuring information quality in the web context: a survey of state-of-the-art instruments and an application methodology. Proceedings of the Seventh International Conference on Information Quality (ICIQ-02).

Recuperado de: http://mitiq.mit.edu/ICIQ/Documents/IQ\%20Conference\%202002/Papers/MeasurelnfoQualityinTheWebContext.pdf

Fink-Shamit, N. \& Bar-Ilan, J. (2008). Information quality assessment on the Web- an expression of behaviour. Information Research, 13(4). Recuperado de: http://InformationR.net/ir/13-4/paper357.html

Lee, Y.W., Strong, D.M., Kahn, B.K. \& Wang, R.Y. (2002). AIMQ: a methodology for information quality assessment. Information \& management 2002 40(2), 133-146. Recuperado de: http://web.cba.neu.edu/ ywlee/publication/aimq.pdf

Peter, O. H. (2001). Information: a question of quality? Scire, 17 (1), 17-21. Recuperado de: http://www.ibersid.eu/ojs/index.php/scire/article/view/4002/3640

Pettigrew, K. E., Fidel, R., \& Bruce, H. (2001). Conceptual frameworks in information behavior. Annual Review of Information Science and Technology (ARIST), 35, 43-78.

Ponjuán, G. (2002). Gestión de información: precisiones conceptuales a partir de sus orígenes. Informação \& Informação, 13, 2638.

Ponjuán, G. (2011). La gestión de información y sus modelos representativos. Valoraciones. Ciencias de la Información, 42 (2), 11 17. 
Rubin, V. L. \& Vashchilko, T. (2012). Extending information quality assessment methodology: a new veracity/deception dimension and its measures. ASIST, (October), 28-31.

Strong, D.M., Lee. Y.W. \& Wang, R.Y. (1997). Data quality in context. Communications of the ACM, 40 (5), 103-110. Recuperado de: http://www.fing.edu.uy/inco/cursos/caldatos/articulos/StrongLeeWangCACMMay97.pdf

Stvilia, B. Gasser, S., Twidale, M. B. \& Smith, L. C. (2007). A Framework for Information Quality Assessment. Journal of the American Society for Information Science and Technology, 58 (12), 1720-1733.

Su Y. \& Jin Z. A methodology for information quality assessment in data warehousing. IEEE International Conference (ICC'08); 2008 mayo. Recuperado de: http://paper.istic.ac.cn/paper/filebase/2006/1215393761242.pdf

Van Birgelen, M., De Ruyter, K. \& Wetzels, M. (2001). What factors determine use of quality-related marketing research information? An empirical investigation. Total quality management, 12 (4), 521-534. Recuperado de: http://arno.unimaas.nl/show.cgi?fid=3188

Zins, C. (2007). Conceptual approaches for defining data, information, and knowledge. Journal of the American Society for Information Science and Technology, 58 (4), 479-493.

\title{
Datos del autor
}

Carlos Luis González-Valiente

Licenciado en Ciencias de lalnformación, especialista en información del Departamento de Informática y Gestión de la Información del Grupo Empresarial de la Industria Sidero-Mecánica - GESIME, Cuba.

carlos.valiente@fcom.uh.cu

\author{
Recibido-Received : $2013-10-29$ \\ Aceptado-Accepted : :2013-12-30
}

\section{(c) EY EY}

This work is licensed under a Creative Commons Attribution 4.0 United States License.

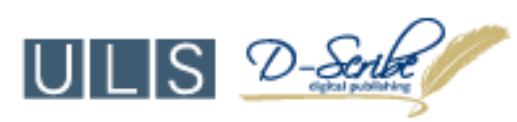

This journal is published by the University Library System of the University of Pittsburgh as part of its $\underline{D}$-Scribe Digital Publishing Program and is cosponsored by the University of Pittsburgh Press. 